\title{
Modélisation numérique des écoulements de laves torrentielles
}

\author{
Dominique Laigle et Philippe Coussot \\ CEMAGREF - GRENOBLE \\ Division Protection Contre les Erosions \\ 2, rue de la papeterie BP.76, 38402 Saint-Martin-d'Hères CEDEX \\ tél. : 76762766
}

Les laves torrentielles se distinguent d'autres écoulements naturels à surface libre [JOHNSON et al. (1984), MEUNIER (1991)] par deux caractéristiques essentielles qui sont :

- la nature du fluide en écoulement qui est constitué d'un mélange d'eau, d'argile et de matériaux granulaires de tailles très variables;

- les caractéristiques de l'écoulement lui-même qui est rapide, transitoire et présente un front raide le plus souvent constitué de blocs.

En première approximation, on néglige l'effet de phénomènes tels que : hétérogénéités de la concentration ou de la granulométrie, glissement, fracturation, érosion, ségrégation ou sédimentation sur l'écoulement d'une lave pleinement développée. Dans ces conditions, nous nous plaçons dans le cadre de la mécanique des fluides homogènes.

L'utilisation des équations de St Venant, ou de formes dérivées, pour modéliser les écoulements transitoires des laves torrentielles est assez fréquente [TAKAHASHI (1987), Mizuyama (1987), Martinet (1992a-b)] et fort peu de tentatives d'emploi d'un autre modèle semblent avoir été menées. Le point de distinction essentiel entre toutes ces utilisations des équations de St Venant porte sur l'expression de la loi de frottement qui découle elle-même des hypothèses concernant la rhéologie des laves torrentielles.

Même si de nombreuses tentatives d'adaptation de modèles rhéologiques ont eu lieu [CHEN (1987)], les hypothèses les plus classiquement retenues sont le modèle de Bingham [JoHnson (1970)] pour les laves boueuses et le modèle de Bagnold [TAKAHASH (1980)] pour les laves granulaires. Des travaux récents tendent à remettre en cause ces hypothèses classiques. DAvies et al. (1991) et Coussot (1992) ont mis en évidence des lois de comportement instables, notamment pour les laves granulaires et Coussot (1992) et MAJOR and PIERSON (1992) ont montré que le modèle rhéologique le mieux adapté aux laves boueuses est celui de Herschel-Bulkley. Partant de cette dernière hypothèse et utilisant le fait que l'écoulement est laminaire, Coussot (1994b-c) a établi une loi de frottement dont les paramètres sont directement déduits de la rhéologie et l'a validée sur des écoulements expérimentaux en régime permanent [Coussot (1994b-d)].

Cette loi de frottement est ici introduite dans un modèle numérique de résolution des équations de St Venant supportant la présence de discontinuités telles qu'un ressaut mobile [VILA (1986) et (1987)]. Nous nous proposons de décrire dans un premier temps les principes et hypothèses du modèle et de la loi de frottement associée. La validation de ce modèle nous amène dans un second temps à le confronter à des écoulements en canal de laboratoire dont les lois de similitude d'échelle ont été préalablement établies [Coussot (1994a)]. De telles tentatives de validation ont été menées pour d'autres modèles mais, contrairement aux résultats ici présentés, elles faisaient

\section{Numerical modelling of debris flow dynamics}

In order to predict complex transient debris flows we developed a numerical model based on shallowwater-equations. We consider only muddy debris flows whose simple shear behaviour is well represented by a Herschel-Buikley model. In laminar regime, the wall friction force is assumed to be equal to the resistance of a steady uniform flow having same depth and mean velocity. The model parameters, deduced from the fluid behaviour, are estimated independently by rheometrical measurements. The main characteristics of our experimental transient flows, established in a laboratory flume, are in fairly good agreement with the numerical model predictions without any additional fitting. 
appel soit à un calage systématique des paramètres [MARTINET (1992a-b)] soit à un calage partiel dans les domaines où une application stricte des hypothèses sousjacentes au modèle rhéologique n'était pas justifiée (utilisation du modèle de Bagnold par TAKAHASH (1987)).

\section{Modélisation des écoulements}

Pour modéliser les laves torrentielles, il nous fallait faire le choix d'un jeu d'équations traduisant les bilans physiques de l'écoulement (conservation de la masse et de la quantité de mouvement) en tenant compte de la présence d'un front, ainsi que le choix d'une loi de comportement traduisant les caractéristiques du matériau.

\subsection{Modélisation mathématique}

Les équations de St Venant peuvent s'écrire sous une forme particulière dite conservative dont l'avantage essentiel est de vérifier la relation de Rankine-Hugoniot (relation de bilan entre l'amont et l'aval d'une discontinuité d'écoulement telle qu'un ressaut pour un écoulement à surface libre). Associée à un schéma numérique approprié, cette formulation permet de construire un modèle numérique capable de traiter la discontinuité comme n'importe quel point courant et d'en déterminer la position à chaque instant, permettant ainsi un traitement global de l'écoulement avec un degré de complexité et des temps de calcul raisonnables [VILA (1986)].

Nous raisonnons sur un écoulement canalisé, traduit par les équations de St Venant monodimensionnelles, qui se mettent alors sous la forme conservative suivante :

$$
\begin{aligned}
\frac{\partial}{\partial t}\left(\begin{array}{l}
S \\
Q
\end{array}\right)+\frac{\partial}{\partial x}\left(\begin{array}{c}
Q \\
\alpha \frac{Q^{2}}{S}+P(S, x)
\end{array}\right)= & \\
& =\left(\begin{array}{cc}
0 \\
g S \sin (\theta)-\text { Frot }+B(S, x)
\end{array}\right)
\end{aligned}
$$

$S \quad$ : section transversale droite de l'écoulement

$Q \quad$ : débit dans cette section

$\alpha \quad$ : coefficient correctif $\left(\alpha=\frac{\overline{u^{2}}}{\bar{u}^{2}}\right)$

Frot : frottement

avec $\quad \frac{\partial P(S, x)}{\partial x}-B(S, x)=g S \cos (\theta) \frac{\partial h}{\partial x}$.

\subsection{Le schéma numérique}

Si l'on considère uniquement le premier membre des équations de St Venant conservatives, en annulant le second membre, on obtient un système strictement hyperbolique non linéaire pour lequel il existe des méthodes bien adaptées de résolution numérique (supportant la présence de discontinuités) telles que le schéma de Godunov que nous utilisons dans le cadre d'une approximation aux volumes finis [VILA (1986) et (1987)]. Une solution de ce problème étant obtenue, une correction est ensuite réalisée pour tenir compte du second membre (gravité, loi de frottement, etc...).

\subsection{La loi de frottement}

Pour un problème où sont connues conditions initiales, conditions aux limites et géométrie du chenal d'écoulement, la loi de frottement reste la seule inconnue des équations de St Venant. Elle seule permet d'introduire le comportement particulier du fluide dans les équations de bilan [CHEN (1987)].

On s'intéresse uniquement au cas des laves torrentielles essentiellement boueuses (fraction argileuse supérieure à $10 \%$ qui ont un comportement en cisaillement simple bien traduit par une loi de type Herschel-Bulkley [COUssot (1992)] :

$$
\begin{aligned}
& \tau=\tau_{\mathrm{c}}+\mathrm{K} \gamma^{\mathrm{n}} \text { avec } \mathrm{n}=1 / 3 \\
& \tau \text { : contrainte de cisaillement } \\
& \tau_{\mathrm{c}}: \text { contrainte seuil } \\
& \gamma: \text { gradient de vitesse de cisaillement } \\
& K \text { et } n \text { : paramètres de la loi }
\end{aligned}
$$

Des considérations théoriques sur l'écoulement d'un fluide de Herschel-Bulkley sur un plan infiniment large, corroborées et complétées par des expériences en canaux de section quelconque ont permis d'établir les expressions suivantes de la contrainte moyenne à la paroi pour un écoulement permanent uniforme dans un canal ouvert [Bossan (1993), Bossan et al. (1993), Coussot (1994b)] :

$$
\tau_{p}=\tau_{c}\left(1+a\left(H_{b}\right)^{-0.9}\right) \quad H_{b}=\frac{\tau_{c}}{K}\left(\frac{h}{U}\right)^{1 / 3}
$$

avec :

- canal plan infiniment large : $\mathrm{a}=1,93$

- canal rectangulaire : $a=1,93-0,43 \arctan \left((10 \mathrm{~h} / \mathrm{L})^{20}\right)$ pour $h / L<1$

L'expression du terme de frottement introduit dans les équations du mouvement est alors donnée par:

Frot $=\frac{\tau_{p}}{\rho} P_{C}$

$P_{t}$ : périmètre mouillé, $\rho$ : masse volumique.

Le frottement en régime permanent tenant compte de la vitesse moyenne et de la hauteur de fluide locales, nous supposons que la résistance locale à l'écoulement transitoire est égale au frottement calculé pour un même débit et une même hauteur d'écoulement locaux par la formule établie en régime permanent uniforme. La même hypothèse faite pour des écoulements graduellement variés a été validée expérimentalement [BosSAN (1993)].

\subsection{Principales hypothèses du modèle}

Les hypothèses de St Venant [CUNGE et al. (1980)] supposent notamment que la densité du fluide est constante et que la courbure des lignes de courant est suffisamment faible pour que l'on puisse négliger les accélérations verticales (vagues raides, fortes variations de pente) ou transversales (accélérations centrifuges, fortes variations de la largeur d'écoulement).

La représentation du front d'écoulement est ici schématisée sur quelques points du maillage par des discontinuités qui vérifient les bilans de masse et de quantité de mouvement mais ne traduisent pas la structure de détail, la longueur ou la raideur exacte du front. 


\section{Validation du modèle}

Pour valider le modèle, on compare ses prédictions à des écoulements de mélanges eau-argile naturelle (considérés comme des fluides de Herschel-Bulkley) réalisés en canal uniforme au laboratoire et en similitude d'écoulements naturels de laves torrentielles [Coussot (1994b)]. Les paramètres de la loi de frottement sont déduits directement de mesures rhéométriques effectuées sur des échantillons des matériaux utilisés ; par conséquent, la confrontation n'introduit pas de calage de paramètres.

\subsection{Les expériences de laboratoire}

Le principe retenu consiste à effectuer, en conditions maîtrisées, des lâchures instantanées d'une quantité donnée de matériau modèle dans un canal et à mesurer les hauteurs d'écoulement en différents points en fonction du temps et cela pour différentes pentes, différentes quantités de matériau lâché, différentes hauteurs initiales et pour des fluides différents.

Les laves torrentielles étant des écoulements par bouffées, deux cas ont été étudiés :

— l'écoulement à hauteur initiale nulle représentatif de la première bouffée ;

- l'écoulement à hauteur initiale non nulle représentatif des bouffées suivantes.

\subsubsection{Procédure}

Les matériaux fabriqués pour les expériences sont constitués de mélanges eau-argile naturelle (Sinard, Isère, France) à différentes concentrations. Ils sont au nombre de quatre notés $\mathrm{A}, \mathrm{B}, \mathrm{C}$ et $\mathrm{D}$ dont les caractéristiques rhéologiques (paramètres de la loi de Herschel-Bulkley) sont reportées dans le tableau suivant:

\begin{tabular}{|c|c|c|c|}
\hline matériau & $\begin{array}{c}\text { masse } \\
\text { volumique } \\
\left(\mathrm{kg} \cdot \mathrm{m}^{-3}\right)\end{array}$ & $\begin{array}{c}\text { contrainte } \\
\text { seuil } \tau_{\mathrm{c}} \\
(\mathrm{Pa})\end{array}$ & $\begin{array}{c}K \text { (modèle de } \\
\text { Herschel-Bulkley) }\end{array}$ \\
\hline A & 1410 & 19,0 & 3,5 \\
\hline B & 1422 & 17,0 & 5,6 \\
\hline C & 1397 & 9,5 & 3,1 \\
\hline D & 1330 & 4,5 & 1,4 \\
\hline
\end{tabular}

Pour plus de détails sur la technique utilisée pour le calage du modèle de Herschel-Bulkley sur les mesures effectuées au rhéomètre, on se reportera à CoussoT (1994a). En cumulant les incertitudes des mesures et du calage de la loi rhéologique, on obtient des erreurs d'estimation de l'ordre de $20 \%$. Le calage a été particulièrement délicat pour le matériau $\mathrm{D}$.

Le dispositif expérimental (fig. 1) est constitué d'un canal à section rectangulaire de $4 \mathrm{~m}$ de long et $0,6 \mathrm{~m}$ de large [BossAN (1993)]. La pente de ce canal est variable (des pentes de $6 \%$ à $31 \%$ ont été utilisées). Son extrémité amont (abscisse $\mathrm{x}=0$ ) est limitée par une cloison. A l'abscisse $0,85 \mathrm{~m}$ est disposée une plaque verticale amovible servant de barrage qui peut être retirée par coulissement rapide vers le haut. Aux abscisses $1,65 \mathrm{~m}, 2,75 \mathrm{~m}$ et $3,85 \mathrm{~m}$, sont disposés 3 capteurs de distance à ultrasons, reliés à une centrale d'acquisition qui enregistre 33 mesures de hauteur d'écoulement par seconde et par capteur et cela pendant 10 secondes.

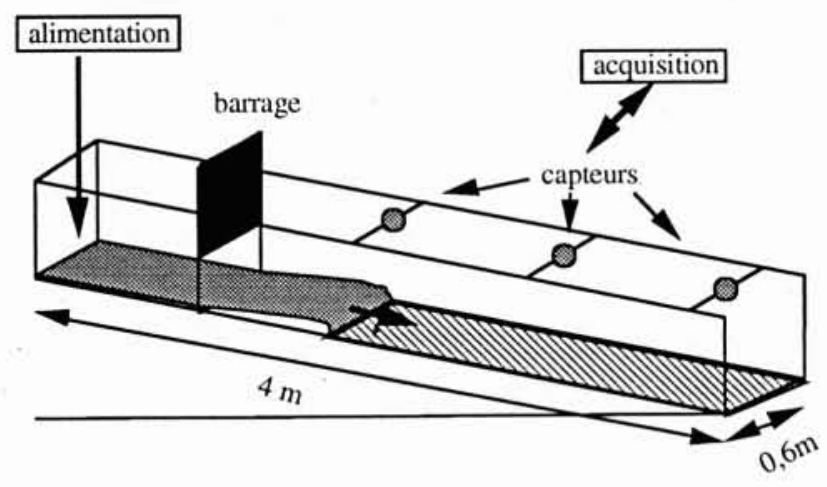

1. Dispositif expérimental

\subsubsection{Les incertitudes expérimentales}

Les incertitudes expérimentales existant sur les mesures et liées, notamment, à la synchronisation entre ouverture du barrage et déclenchement de l'acquisition, au principe de fonctionnement des capteurs à ultrasons ou au fonctionnement du dispositif d'ouverture du barrage ont été estimées de l'ordre de 5 à $15 \%$ des valeurs mesurées de hauteurs ou de temps de parcours.

\subsection{Ecoulements à hauteur initiale nulle}

\subsubsection{Comportement et sensibilité du modèle}

Afin d'obtenir une estimation a priori du comportement du modèle et de sa sensibilité dans la gamme des essais expérimentaux réalisés, nous avons calculé la réponse de ce dernier en termes de hauteurs maximales et temps de parcours pour une plage de variation des paramètres recouvrant bien les conditions expérimentales testées.

En référence aux conditions expérimentales, nous nous placons dans le cas d'écoulements en canal supposé très large et présentant deux particularités importantes :

- la vitesse initiale de l'écoulement est nulle en tous points ;

- la hauteur initiale d'écoulement dans le canal est nulle et la hauteur stockée derrière le barrage est à surface libre horizontale, les conditions initiales pour un matériau et une pente données sont alors entièrement fixées par la hauteur de barrage (Hbar).

La mise sous forme adimensionnelle des équations de $\mathrm{St}$ Venant permet de mettre en évidence deux nombres sans dimension qui, dans les conditions précédemment énon- 
cées, déterminent complètement l'écoulement en tout point du canal :

$$
G^{\prime}=\frac{\rho g \mathrm{Hbar} \sin (\theta)}{\tau_{c}} \quad H^{\prime}=\frac{K}{\tau_{c}}\left(\frac{g \cos (\theta)}{\mathrm{Hbar}}\right)^{n / 2}
$$

Hbar: hauteur au niveau du barrage à $\mathrm{t}=0$

Les essais expérimentaux se situent dans une gamme de variation de 4 a 70 pour le paramètre $G^{\prime}$ et de 0,35 à 0,80 pour le paramètre $H^{\prime}$. Cette fourchette de valeurs est suffisamment large pour inclure des écoulements en similitude de la plupart des laves réelles. Par exemple, le produit $\mathrm{A}$ en écoulement dans le canal de $4 \mathrm{~m}$ de longueur traduit à l'échelle $1 / 30$ le comportement d'un matériau naturel ( $\rho=2200 \mathrm{~kg} \cdot \mathrm{m}^{-3}, \tau_{\mathrm{c}}=900 \mathrm{~Pa}, K=290$ environ) dans un chenal de $120 \mathrm{~m}$ de long.

Pour un écoulement pleinement développé, après un abaissement rapide observé lors de la mise en vitesse, les hauteurs maximales tendent asymptotiquement vers une valeur quasi-constante (fig. 2 et 3 ). Les vitesses du front restent à peu près constantes au cours de ces deux phases. L'arrêt se traduit par une diminution rapide des hauteurs maximales d'écoulement et des vitesses de propagation du front (fig. 2 et 3 ).

Le modèle présente une sensibilité faible pour un écoulement pleinement développé, mais forte à proximité d'une phase d'arrêt (fig. 2 et 3 ). Les résultats présentés, établis pour une variation de $G^{\prime}$, sont qualitativement valables pour une variation de $H^{\prime}$. Pour un écoulement pleinement développé, la sensibilité diminue pour une diminution de $H^{\prime}$ ou une augmentation de $G^{\prime}$.

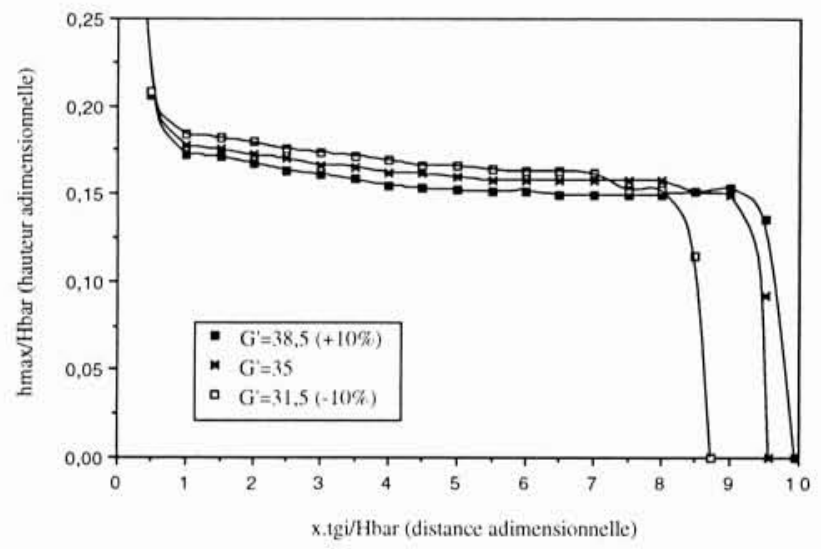

2. Sensibilité sur les hauteurs maximales dëcoulement $\left(H^{\prime}=\right.$ 0,684 )

\subsubsection{Confrontation entre expériences et simulation.}

\subsubsection{Régime permanent}

Des comparaisons effectuées en régime permanent non uniforme ont montré une excellente concordance entre les courbes de remous établies numériquement et celles calculées directement à partir de la loi de frottement. Ces dernières s'avèrent par ailleurs capables de décrire les

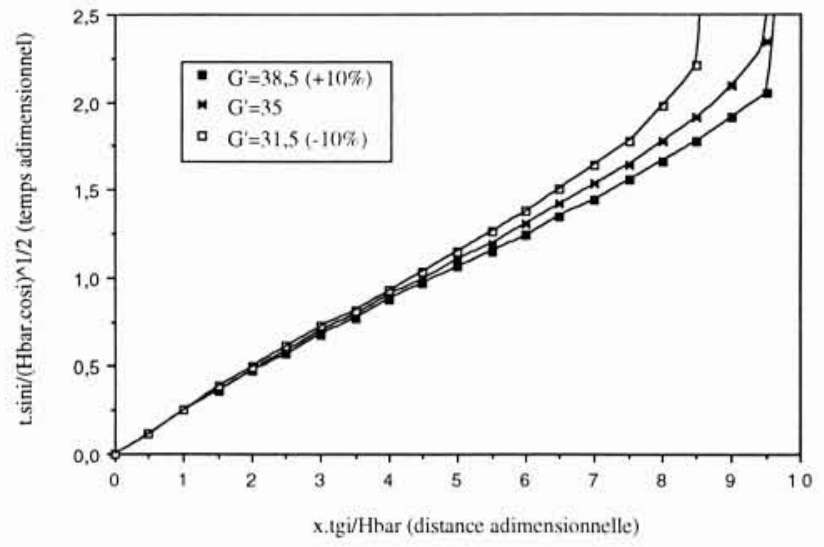

3. Sensibilité sur les temps de parcours $\left(H^{\prime}=0,684\right)$

données expérimentales avec une incertitude inférieure à $25 \%$ [Bossan (1993)].

\subsubsection{Ecoulements transitoires}

Qualitativement, les simulations et les mesures présentent une bonne concordance ( fig. 4). La forme des hydrogrammes est généralement bien respectée, les ordres de grandeur des hauteurs et des temps de propagation sont tout à fait cohérents avec les mesures.

Les différences portent sur :

— un décalage des hauteurs maximales d'écoulement dans un rapport généralement inférieur à $\pm 20 \%$ de la valeur mesurée: seulement $12 \%$ des valeurs calculées s'écartent de cette fourchette, un certain nombre d'entre elles correspondent à des phases d'arrêt (fig. 5) pour lesquelles nous avons vu que la sensibilité est forte:

— un décalage du temps d'arrivée au niveau d'un capteur généralement compris dans la fourchette - $20 \%$ à $+10 \%$ de la valeur mesurée (fig. 6) sauf dans le cas d'écoulements proches d'une phase d'arrêt pour lesquelles le décalage peut être important (sensibilité forte);

- un décalage de la hauteur d'écoulement observée en fin d'expérience (à $t=10$ secondes) pouvant varier entre 0 et +10 à $+15 \%$ de la hauteur mesurée ;

- dans le cas de calculs de distances d'arrêt, les différences atteignent $\pm 25 \%$ de la distance parcourue mesurée ;

L'analyse des résultats ne permet pas de mettre en évidence de liaison claire entre incertitudes d'une part et nombres adimensionnels $\left(G^{\prime}\right.$ ou $\left.H^{\prime}\right)$ ou échelle géométrique des essais d'autre part. Les plus grosses incertitudes apparaissent pour le matériau $D$ dont les paramètres rhéologiques ont été très délicats à déterminer. La plupart des différences observées restent à l'intérieur de la fourchette de précision que l'on peut raisonnablement attendre des mesures. Les incertitudes sur l'estimation des paramètres rhéologiques ou les incertitudes expérimentales en général sont assez fortes pour expliquer ces différences et masquer les imprécisions du modèle lui-même. 
pente $21 \%$ Hbarrage $14 \mathrm{~cm}$ matériau A

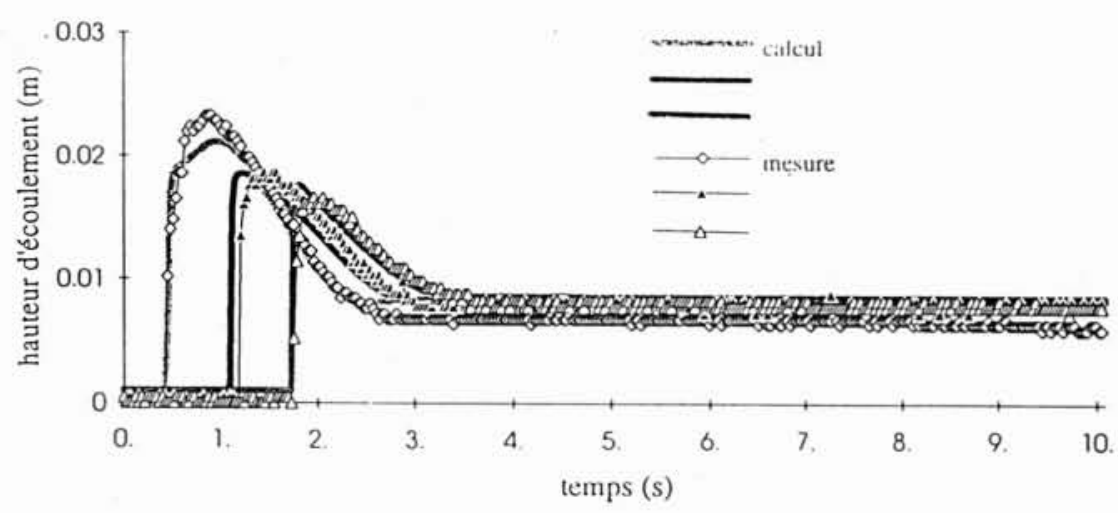

4. Exemple de comparaison simulations/mesures (hauteur initiale nulle)

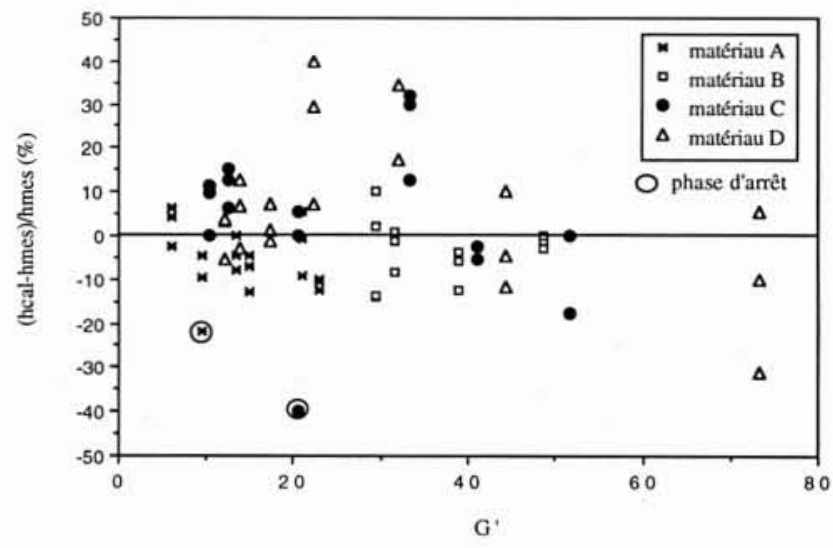

5. Comparaison simulations/mesures sur les hauteurs maximales d'écoulement

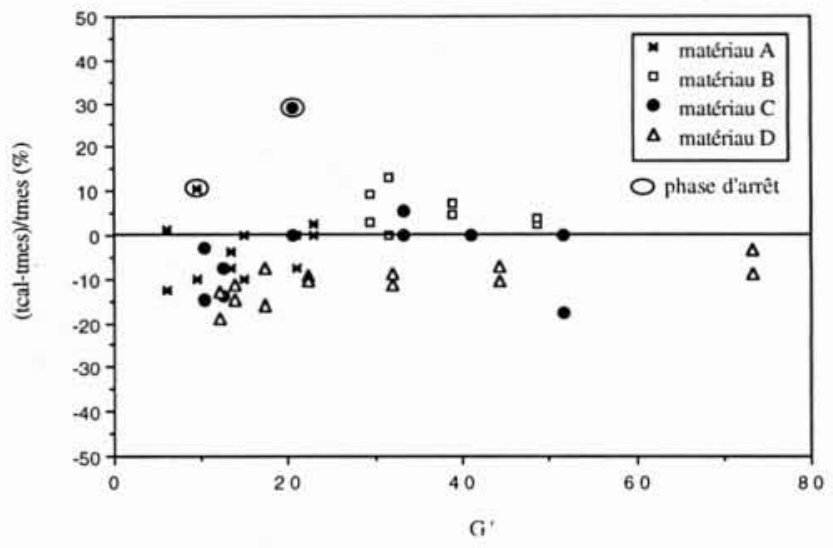

6. Comparaison simulations/mesures sur les temps de parcours

pente $16 \%$ Hbarrage $9 \mathrm{~cm}$ matériau A

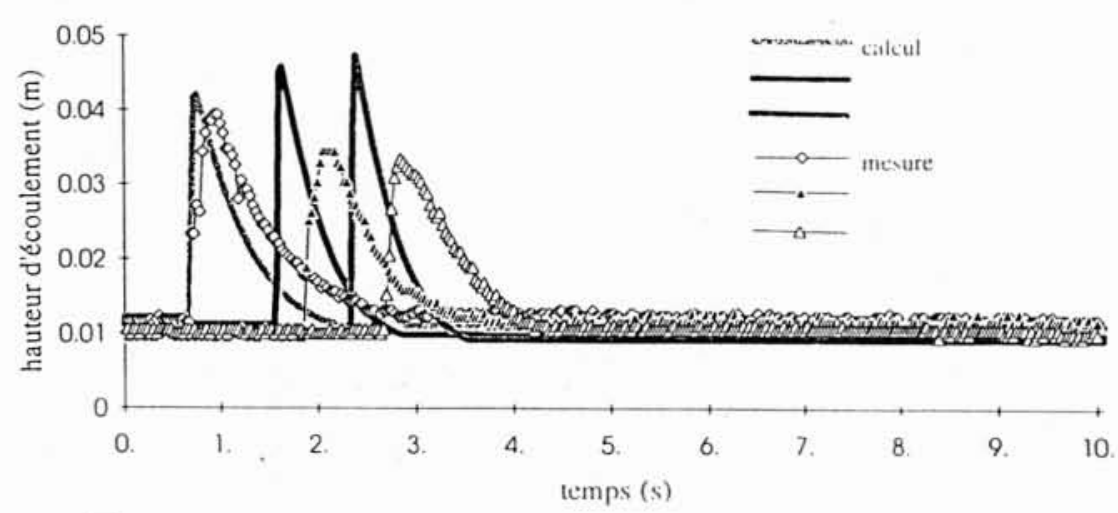

7. Exemple de comparaison simulations/mesures (hauteur initiale non nulle)

\subsection{Ecoulements à hauteur initiale non nulle}

Les vagues calculées peuvent être plus raides, plus rapides, avec une pointe plus marquée et une phase de tarissement plus courte que les vagues mesurées. L'amplitude des différences, qui peut atteindre $+70 \%$ des hauteurs maximales et $-35 \%$ des temps d'arrivée mesurés aux capteurs (fig. 7) évolue nettement avec l'amplitude des vagues. Le critère pertinent permettant de distinguer les cas d'une bonne ou d'une mauvaise concordance entre mesures et calculs (fig. 8), à l'échelle des essais réalisés, est le rapport entre la hauteur de barrage et la hauteur de matériau initialement présente dans le canal. Ce critère remplace le paramètre $G^{\prime}$ (établi pour une hauteur initiale nulle), sa signification en est physiquement très proche dans le cas de nos essais. Dans tous les cas traités expérimentalement, 


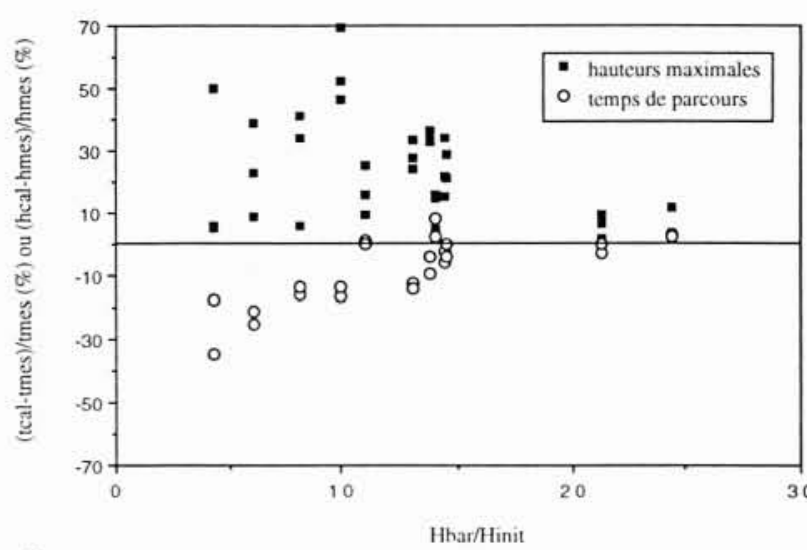

8. Comparaison simulations/mesures sur les hauteurs maximales d'écoulement et les temps de parcours (hauteur initiale non mulle)

l'amplitude des vagues, ả l'échelle des essais réalisés, reste relativement constante au cours du déplacement.

Une interprétation possible de cette différence tient à l'incapacité du modèle à traduire certains types de vagues [STOKER (1957)] présentant des accélérations verticales non négligeables mais qui ne peuvent être assimilées au déplacement d'un front. C'est le cas par exemple des ondes sinusoīdales, cnoïdales ou solitaires [NAAIM (1991)].

Une seconde interprétation tient à la difficulté de traduire correctement la mise en mouvement et l'incorporation du matériau présent dans le canal à l'écoulement derrière le front compte tenu des effets transitoires engendrés et de la modification du profil des vitesses par rapport aux hypothèses.

\section{Conclusion}

La modélisation adoptée montre de bonnes capacités à prédire les écoulements des mélanges eau-argile étudiés. Le bien-fondé d'une utilisation de la loi de frottement proposée, en régime transitoire, est établi.

A condition de veiller à rester dans le domaine de validité mis en évidence (rapport Hbar/Hinit supérieur à environ 20), il est vraisemblable que le modèle proposé introduit des erreurs suffisamment faibles pour être masquées par l'amplitude des incertitudes expérimentales (notamment sur l'estimation des paramètres rhéologiques). Dans l'état actuel des recherches, les efforts doivent porter essentiellement sur la compréhension de phénomènes physiques encore imparfaitement élucidés, leur description et leur quantification fines et la validation des modèles construits par confrontation a l'expérience. La démarche présentée se prête parfaitement à ce type de validation.

Parallèlement, le modèle doit encore évoluer pour permettre d'aborder des cas de figures plus proches du terrain (géométrie plus complexe, prise en compte spécifique du front rocheux, matériaux plus granulaires). Bien qu'une utilisation systématique de type ingénierie soit encore prématurée, le modèle constitue déjà un bon outil pour tester schématiquement l'influence d'hypothèses physiques sur des écoulements transitoires. Une version bidimensionnelle capable de traiter les phénomènes d'éta- lement des laves (écoulement sur un cône de déjections par exemple) et fondée sur le même principe est en cours d'établissement.

Les résultats prometteurs de ce modèle permettent d'envisager son utilisation pour d'autres écoulements naturels transitoires (éboulements, avalanches, laves volcaniques, etc...) à partir du moment où dans les mêmes conditions que pour les laves torrentielles, il sera possible de déterminer la loi de frottement à partir de connaissances concernant leur loi de comportement.

\section{Références}

BosSAN E. (1993). - Expériences d'écoulements de laves torrentielles sur modèle réduit DEA de mécanique, INPG, Grenoble, France.

Bossan E., Krage J.-U. et Tigerghien G. (1993). - Hydraulique des laves torrentielles - Expériences d'écoulement à surface libre en canal ouvert - . Projet de fin d'études de l'ENSHMG. $100 \mathrm{p}$.

CHEN C.L. (1987). - Comprehensive review of debris flow modeling concepts in Japan, Geological Society of America, Reviews in Engineering Geology, vol. VII, pp. 13-29.

Coussot P. (1992). - Rhéologie des laves torrentielles, thèse do Doctorat de l'Institut National Polytechnique de Grenoble, Grenoble, France, 420 p.

Coussot P. (1994a). - Rhéologie des laves torrentielles - La Houille Blanche $n^{\circ} 3 / 94$

Coussot P. (1994b). — Lois découlement des laves torrentielles La Houille Blanche $n^{\circ} 3 / 94$.

Coussot P. (1994c). - Steady, laminar, flow of concentrated mud suspensions in open channel - I theory - submitted to Journal of Hydraulic Research.

Coussot P. (1994d). - Steady, laminar, flow of concentrated mud suspensions in open channel - II experiments and comparison with theory: uniform flows - submitted to Journal of Hydraulic Research.

Cunge J.A.. Holly F.M., Verwey A. (1980). - Practical Aspects of Computational River Hydraulics, Pitman, London, England.

Davies T.R., Phillips C.J., Pearce A.J., Zhang Xin Bao (1991). - New aspects of debris flow behaviour, Japan-U.S. Symposium on Snow Avalanche, Landslide, Debris Flow Prediction and Control.

Johnson A.M. (1970). - Physical processes in Geology, W.H Freeman, San Francisco, 577 ,

JoHnSON A.M. and Rodine J.R. (1984). - Debris flows, in : Slope Instability, (D. Brunsden and D.B. Prior Editors), chapter 8, John Wiley and sons, New-York.

MAJOR J.J. and PIERSON T.C. (1992). - Debris flow rheology: experimental analysis of fine-grained slurries. Water Ressources Research, vol, 28, n³, pp. 841-857.

MARTINet G. (1992a), - Contribution à la modélisation des avalanches de neige dense et des laves torrentielles, thèse de Doctorat de I'Université Joseph Fourier, Grenoble, France, $218 \mathrm{p}$.

MARTINet G. (1992b). - Modélisation tridimensionnelle des laves torrentielles - Application à un cas réel: Utilisation du code 
bidimensionnel LAVEID-, Colloque INTERPRAEVENT 1992, Berne, Suisse, vol. II, pp. 331-342.

MEUNIER M. (1991). - Eléments d'hydraulique torrentielle, CEMAGREF, Collection Etudes, série Montagne n 1, 274 p.

Mizuyama T. and YaZawa T. (1987). - Computer simulation of debris flow depositional processes, Erosion and sedimentation in the Pacific Rim (Proceedings of the Corvallis Symposium), IAHS n ${ }^{\circ} 165$, pp. 179-190.

NAAIM M. (1991). - Modélisation numérique des effets hydrodynamiques provoqués par un glissement solide dans une retenue. thèse de Doctorat de l'Université Joseph Fourier, Grenoble, France, $157 \mathrm{p}$.

StOKer J.J. (1957). - Water waves, Interscience, New York.
TAKAhASHI T. (1980). - Debris flow on prismatic open channel, Journal of the Hydraulics Division, 106(HY3), pp. 381-396.

Takahashi T., Nakagawa H. et Kuang S. (1987). - Estimation of debris flow hydrograph on varied slope bed. Erosion and sedimentation in the Pacific Rim (Proceedings of the Corvallis Symposium), IAHS n`165, pp. 167-177.

VILA J.P. (1986). - Sur la théorie et l'approximation numérique de problèmes hyperboliques non linéaires, applications aux équations de Saint Venant et à la modélisation des avalanches de neige dense, thèse de Doctorat de I'Université Paris VI, Paris, France, $481 \mathrm{p}$.

VILA J.P. (1987). - Schémas numériques en hydraulique des écoulements avec discontinuités, Actes du colloque AIRH, Lausanne, Suisse. 